\title{
Giant disseminated condylomatosis in SLE
}

L CostaPinto, MFR Grassi, K Serravalle, ACV Travessa, VNO Olavarria and MB Santiago Lupus 2012 21: 332 originally published online 30 September 2011

DOI: $10.1177 / 0961203311421207$

The online version of this article can be found at:

http://lup.sagepub.com/content/21/3/332

\author{
Published by: \\ (SAGE \\ http://www.sagepublications.com
}

Additional services and information for Lupus can be found at:

Email Alerts: http://lup.sagepub.com/cgi/alerts

Subscriptions: http://lup.sagepub.com/subscriptions

Reprints: http://www.sagepub.com/journalsReprints.nav

Permissions: http://www.sagepub.com/journalsPermissions.nav

>> Version of Record - Feb 3, 2012

OnlineFirst Version of Record - Sep 30, 2011

What is This? 


\title{
CASE REPORT
}

\section{Giant disseminated condylomatosis in SLE}

\author{
L CostaPinto $^{1,2}$, MFR Grassi ${ }^{3}$, K Serravalle $^{4}$, ACV Travessa $^{2}$, VNO Olavarria $^{3}$ and MB Santiago ${ }^{1}$ \\ 'Escola Bahiana de Medicina e Saúde Pública, Salvador, Brazil; ${ }^{2}$ Departamento de Obstetrícia e Ginecologia, Universidade Federal da Bahia, \\ Brazil; ${ }^{3}$ Centro de Pesquisas Gonçalo Moniz/Fundação Oswaldo Cruz, Salvador, Brazil; and ${ }^{4}$ Laboratório Silvany Studart, Salvador, Brazil
}

\begin{abstract}
Introduction: Females with systemic lupus erythematosus (SLE) have higher prevalence of human papillomavirus (HPV) infection, which can lead to the development of warts. Herein we report the first case of giant disseminated condylomatosis (GDC) in a SLE female on mycophenolate mofetil (MMF). Case report: The patient, a 33-year-old, Black female, was diagnosed with SLE during her first pregnancy in 2003 based on the features of arthritis, skin rash, seizures, nephritis and presence of antinuclear antibodies. Her pregnancy resulted in preterm delivery of a stillborn fetus at 28 weeks. Since that time she has been treated with steroids and different regimens of immunosuppressive drugs such as cyclophosphamide, azathioprine and lately MMF. In the last few years she presented GDC involving the genital area in addition to skin on the lower abdomen. Topical therapy with trichloroacetic acid, imiquimod and podophyllin was only partially effective. Different types of HPV were identified in the lesions, being HPV-11 in abdomen, HPV 6, 11, 42 in vulva, HPV-6, 11 in vagina and HPV-6, 11 in endocervix. Conclusions: GDC may be a complication of SLE, secondary to the disease itself, its treatment or other factors not yet identified. Lupus (2012) 21, 332-334.
\end{abstract}

Key words: giant disseminated condylomatosis; human papillomavirus (HPV); immunosuppressants; systemic lupus erythematosus; warts

\section{Introduction}

Patients with systemic lupus erythematosus (SLE), particularly those on immunosuppressive therapy, have higher prevalence of human papillomavirus (HPV) infection. ${ }^{1}$

HPV is responsible for various diseases in the anogenital region, varying from benign condyloma acuminatum to carcinoma. The types 6 and 11 are associated with genital warts and cases of lowgrade squamous intraepithelial lesions. Fifteen HPV types are considered high-risk oncogenic (most frequently 16, 18, 33, 35, 45, 52 and 58), as they are associated with high-grade squamous intraepithelial neoplasia and invasive cancers. ${ }^{2}$ This virus can cause giant condyloma acuminatum, also known as Buscheke-Lowenstein tumor (BLT). It is typically a histologically benign disease, but it

Correspondence to: Mittermayer B Santiago, Escola Bahiana de Medicina e Saúde Pública, Rua Frei Henrique, 08, 40.050-420 Salvador, Bahia, Brazil

Email: mitter@svn.com.br

Received 24 February 2011; accepted 28 July 2011 may clinically present as a malignant disease due to its expansive and invasive growth. ${ }^{3}$ Although, malignant transformation in the course of the BLT occurs in up to $50 \%$ of the cases, distant metastases are rare. ${ }^{4}$

Herein we report the first case of giant disseminated condylomatosis (GDC) in a SLE female during the course of mycophenolate mofetil (MMF) therapy.

\section{Case report}

The patient, a 33-year-old Black female was diagnosed with SLE during her first pregnancy in 2003 based on the features of arthritis, discoid rash, seizures, nephritis and presence of antinuclear antibodies, in addition to a skin biopsy revealing features of lupus. Her pregnancy resulted in preterm delivery of a stillborn fetus at 28 weeks. Her first sexual intercourse was at the age of 26 years and she has had only one partner since that time. She had menarche at 14 years of age, and her menstrual cycles 
have been irregular. Since that time she has been treated with steroids as well as different regimens of immunosuppressive drugs such as cyclophosphamide, azathioprine and lately MMF (2g daily). Genital warts first appeared in 2004 and low grade intraepithelial lesion (LSIL) was detected by cytology, and cervical intraepithelial neoplasia (CIN 2) and vulvar intraepithelial neoplasia (VIN 2) were detected by histology. Initially, she was successfully treated with trichloroacetic acid at $70 \%$ in the vulva and electrosurgical excision procedure with a loop (LEEP) in the cervix. After seven months on MMF therapy in 2009 for massive proteinuria, genital warts recurred in the vulva, vagina and perianal area. At that time, she was accidentally burned with hot water (second and third grade), leaving scars on the lower abdomen, pubic mound and lower and medium thighs. The burn scars became progressively hypertrophic, resembling cauliflower, spreading to the lower abdomen, vulva and thighs, vagina, vulva, perineum and anal region (Figure 1, A and B). Biopsies were performed in these locations showing acanthosis, papillomatosis and hyperkeratosis. Endocervical cytology showed high grade intraepithelial lesion (HSIL) and cervical biopsy demonstrated condyloma. Different types of HPV were identified in the lesions, being HPV-11 in the abdomen, HPV $6,11,42$ in the vulva, HPV-6, 11 in the vagina and HPV-6, 11 in the endocervix by PapilloCheck assay. ${ }^{5}$ A search for HPV in the endocervical smear was also positive by in-house PCR as described previously. ${ }^{6}$ Co-infection with HIV, HTLV-1, syphilis and hepatitis B and $\mathrm{C}$ was ruled out by serologic tests. Therapy with trichloroacetic acid, TLR7 agonist imiquimod 5\% cream and later with podophyllin $2 \%$ oil resulted in only partial improvement. Recently she was submitted to a surgical procedure and showed a reasonable response (Figure 1C).

\section{Discussion}

HPV is estimated to be the most common sexually transmitted infection in the US population. ${ }^{2}$ Young age (14 to 24 years) and sexual behavior (multiple sexual partners, increased frequency of intercourse) are the two major factors in the incidence of genital HPV infection. ${ }^{2}$

In a recent systematic review we demonstrated a higher prevalence of HPV infection in SLE patients. ${ }^{1}$ Except for immunosuppression (secondary to the treatment and/or the disease itself), most other known risk factors for HPV infection were absent in the present case. We have no convincing explanation for why our patient developed such an unusual form of condylomatosis (GDC). Although her immunosuppression could partially justify such a complication, ${ }^{7}$ as observed in patients with $\mathrm{HIV}^{3,8,9}$ and kidney transplant recipients (KTRs) on an immunosuppressive regimen, ${ }^{10}$ in her case, it does not exclusively seem to be the case, as taking immunosupressive drugs is a commonplace in SLE and GDC, and has not been previously been observed in this disorder. Curiously, it has been suggested that in KTRs at least, cutaneous warts are more common in those taking azathioprine $(20.6 \%)$ than in those on $\operatorname{MMF}(3.5 \%))^{10}$ The influence of any particular immunosuppressive drug in the present case is difficult to assess as she has been exposed to different types of these drugs. The types of HPV found in her lesions (HPV 6 and 11) are in agreement with the descriptions given in other cases of GDC not associated with SLE. It is notable that the development of warts in areas of burn scars is unique, and no similar description could be found in the literature.

In conclusion, although rare, GDC may be a complication of SLE, secondary to the disease itself, its treatment or other factors not yet identified.

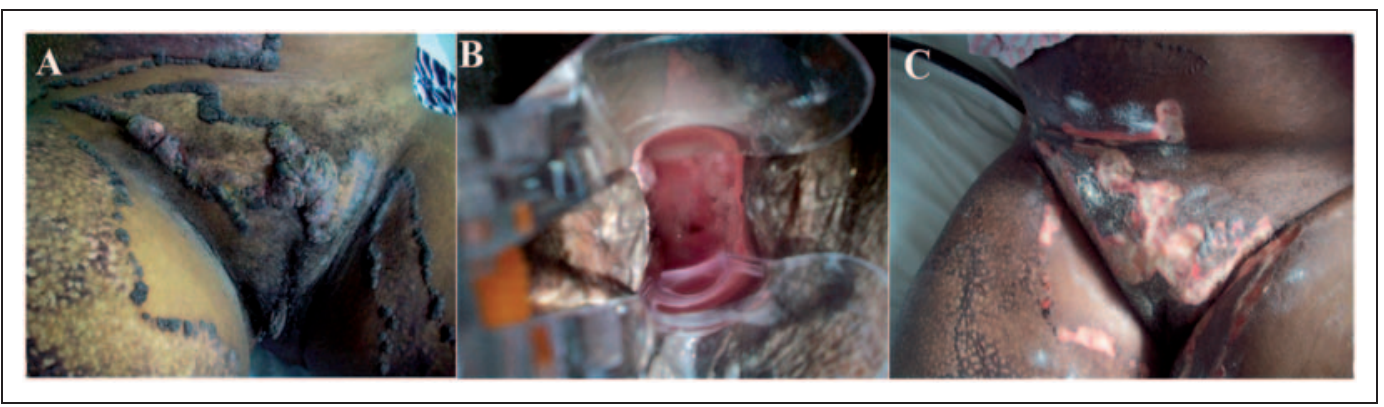

Figure 1 Giant disseminated condylomatosis in patient with systemic lupus erythematosus during mycophenolate mofetil therapy. Disseminated warts on the lower abdomen, vulva, thigh (A) and vaginal wall (B) did not respond to conservative treatment with trichloracetic acid, imiquimod and podophyllin. (C) Aspect of lesions 8 days after surgical resection. 


\section{Acknowledgment}

We are grateful to Dr Conceição Maria Passos de Queiroz and Sérgio Arruda for the histologic analysis.

\section{Funding}

This work was supported by the Conselho Nacional de Desenvolvimento Científico e Tecnológico (CNPq) and FIDEPS (to MS).

\section{Conflict of interest statement}

The authors have no conflict of interest that is directly relevant to the content of this manuscript.

\section{References}

1 Santana IU, Gomes AD, Lyrio LD, Rios Grassi MF, Santiago MB. Systemic lupus erythematosus, human papillomavirus infection, cervical pre-malignant and malignant lesions: a systematic review. Clin Rheumatol, 2011; 30(5): 665-672.

2 Sweet RL, Gibbs RS. Human Papilloma Virus. Infectious diseases of the female genital tract, 5th ed. Philadelphia, PA: Lippincott Williams and Wilkins; 2009; p. 80-94.

3 Mudrikova T, Jaspers C, Ellerbroek P, Hoepelman A. HPV-related anogenital disease and HIV infection: not always 'ordinary' condylomata acuminata. Neth J Med 2008; 66: 98-102.

4 Wietfeldt ED, Thiele J. Malignancies of the anal margin and perianal skin. Clin Colon Rectal Surg 2009; 22: 127-135.

5 Jones J, Powell NG, Tristram A, Fiander AN, Hibbitts S. Comparison of the PapilloCheck DNA micro-array Human Papillomavirus detection assay with Hybrid Capture II and PCR-enzyme immunoassay using the GP5/6+ primer set. $J$ Clin Virol 2009; 45: 100-104.

6 Rabelo-Santos SH, Levi JE, Derchain SF, et al. DNA recovery from Hybrid Capture II samples stored in specimen transport medium with denaturing reagent, for the detection of human papillomavirus by PCR. J Virol Methods 2005; 126: 197-201.

7 Korkmaz C, Urer SM. Cutaneous warts in patients with lupus erythematosus. Rheumatol Int 2004; 24: 137-140.

8 Gonzalez LR, Bueno SG, Garcia NR, Maganto PE, Garcia GR, Escudero BA. [Severe condylomatosis treated with $\mathrm{CO} 2$ laser]. Actas Urol Esp 2006; 30: 845.

9 Sobhani I, Vuagnat A, Walker F, et al. Prevalence of high-grade dysplasia and cancer in the anal canal in human papillomavirusinfected individuals. Gastroenterology 2001; 120: 857-866.

10 Dicle O, Parmaksizoglu B, Gurkan A, Tuncer M, Demirbas A, Yilmaz E. Choice of immunosuppressants and the risk of warts in renal transplant recipients. Acta Derm Venereol 2008; 88: 294-295. 